\title{
ESTIMACIÓN DE COMPONENTES DE VARIANZA Y PREDICCIÓN DE VALORES GENÉTICOS EN POBLACIONES DE ACACIA AZUL USANDO EL ALGORITMO DE CADENAS INDEPENDIENTES
}

\author{
Freddy Mora', Sandra Perret ${ }^{2}$, Carlos Alberto Scapim ${ }^{3}$ \\ Elias N. Martins ${ }^{4}$ y Maria Paz Molina ${ }^{5}$
}

\section{RESUMEN}

Diversos estudios han enfatizado las ventajas de la utilización de los métodos de estimación y predicción basados en Máxima Verosimilitud Restringida (REML) y la Mejor Predicción Linear Insesgada (BLUP) en el análisis genético de especies forestales. Por otra parte, existen otras metodologias que permiten la obtención también robusta, tanto de los componentes de varianza como la predicción de valores genéticos, como por ejemplo la inferencia Bayesiana basada en los algoritmos Markov Chain Monte Carlo (MCMC). El presente estudio busca examinar la viabilidad de la obtención de parámetros genéticos y valores predichos en el mejoramiento genético de especies forestales, usando una variante de la metodologia MCMC llamado algoritmo de Cadenas Independientes ( $\mathrm{CI}$ ). Para ilustrar el análisis genético Bayesiano se usó un ensayo de procedencias de Acacia saligna, establecido en el norte de Chile, en el marco de un proyecto financiado por el Fondo de Desarrollo e Innovación (FDI) y desarrollado por el Instituto Forestal (INFOR). El diámetro medio de copa fue analizado en cada genotipo en un diseño de bloques completos al azar. Las estimaciones de los componentes de varianza y la predicción de los valores genéticos vía $\mathrm{Cl}$ fueron comparadas con los procedimientos clásicos de estimación/predicción REML/ BLUP. En el caso Bayesiano, las distribuciones a posteriori de los parámetros se caracterizaron a través del promedio, la mediana y la moda de cada muestra $\mathrm{Cl}$ a posteriori de tamaño 11000. Componentes de varianza y correlaciones intraclase fueron muy similares entre ambas metodologías; tal correlación tuvo un valor de $27,33 \%$ (REML) y de $26,99 \%-28,48 \%$ (IC). BLUP y la predicción Bayesiana entregaron valores genéticos similares; no se evidenciaron cambios en el ordenamiento de los genotipos (ranking) entre un procedimiento y otro. Ambas metodologias apuntan como las mejores procedencias a Greenough River, Lancelin y Ravensthorpe 2. Se evidenció una alta asociación entre REML/BLUP y Cl, con ello los autores recomiendan ampliamente la utilización de inferencia Bayesiana en el análisis genético de especies perennes.

Palabras clave: Cadenas Independientes, Bayes, REML/BLUP, modelos mixtos.

\footnotetext{
' Departamento de Agronomia, Centro de Ciências Agrárias, Universidade Estadual de Maringá-Paraná, Brasil morapoblete@gmail.com

${ }^{2}$ Instituto de Investigación Forestal, INFOR. sperret@infor.cl

${ }^{3}$ Departamento de Agronomia, Centro de Ciências Agrárias, Universidade Estadual de Maringá-Paraná. Brasil. cascapim@uem.br

4 Departamento de Zootecnia. Centro de Ciências Agrárias, Universidade Estadual de Maringá-Paraná, Brasil. enmartins@uem.br

sInstituto de Investigación Forestal, INFOR. mmolina@infor.cl
} 


\section{ESTIMATION OF VARIANCE COMPONENTS AND PREDICTION OF BREEDING VALUES IN BLUE-LEAF WATTLE POPULATIONS BY USING INDEPENDENCE CHAIN ALGORITHM}

\section{SUMMARY}

Several studies have emphasized the advantages of Best Linear Unbiased Predictor method (BLUP) for the genetic analysis of perennial species, and the Restricted Maximum Likelihood principle (REML) for the estimating of variance components. On the other hand, there are others methods that allow robust estimates of the variance components and the prediction of breeding values, i.e. Bayesian framework: inference based on Markov Chain Monte Carlo algorithms (MCMC). The present study aimed to examine genetic parameters, including the prediction of breeding values, by using Independence Chain algorithm (IC), a variant of the MCMC method. A provenance trial of Acacia saligna, localized in northern Chile, was used to illustrate the Bayesian approach. This field trial was established by Instituto Forestal (INFOR), which is carrying out a collaborative project sponsored by the Fondo de Desarrollo e Innovación (FDI). Crown diameter was analyzed in each genotype considering a randomized complete block design. The estimates of variance components and the prediction of breeding values - based in the Bayesian context, were compared with the classic procedures REML/BLUP. In the Bayesian case, the posterior distributions of the parameters were characterized by the mean, median and mode, which were generated from samples IC with size 11000. The Bayesian and REML approaches provided very similar estimates of variance components and intra-class correlation coefficients; the intra-class correlations were $27.33 \%$ (REML) and $26.99 \%-28.48 \%$ (IC). BLUP and the Bayesian prediction provided similar breeding values; no change was evidenced between the ranking REML/BLUP and IC. The provenances Greenough River, Lancelin and Ravensthorpe 2 were the best, determined by both prediction methods. It was evidenced a high association (correlation) between REML/BLUP and IC, so, we suggest that the Bayesian inference (implemented with the independence chain algorithm) may be broadly used in the genetic evaluation of forest trees.

Keywords: Independence Chain, Bayes, REML/BLUP, mixed models. 


\section{INTRODUCCION}

Numerosos trabajos han mostrado que el método de la Máxima Verosimilitud Restringida (REML; del inglés: Restricted Maximum Likelihood), para la estimación de componentes de varianza, es actualmente el procedimiento idóneo para la obtención del Mejor Estimador Lineal Insesgado (BLUE; del inglés: Best Linear Unbiased Estimator) y de la Mejor Predicción Lineal Insesgada (BLUP; del inglés: Best Linear Unbiased Prediction) en modelos lineales mixtos, con datos de observaciones fenotipicas no balanceado, cuyas estimativas no son sesgadas.

Perri y lemma (1999) mencionan que en el método REML, la función de verosimilitud es dividida en dos partes independientes, una referente a los efectos fijos y otra a los efectos aleatorios, de manera que la función de verosimilitud es dada por la suma de las funciones de verosimilitud de cada parte. El método REML supone normalidad, es iterativo y entrega siempre estimaciones no negativas de los componentes de varianza. Además, este método considera la pérdida de grados de libertad debido a los efectos fijos, entregando estimadores insesgados y de varianza minima para datos balanceados.

Para la obtención de BLUE, que es la estimación de los efectos fijos, y de BLUP, particularmente relacionados al valor genético, se debe conocer los componentes de varianza asociados al modelo (experimento), los cuales deben ser estimados con la mejor precisión posible (Mora et al., 2007a). Los componentes de varianza vía REML pueden ser obtenidos por medio de los procedimientos MIXED y VARCOMP de SAS® (Astorga y Mora, 2005; Mora y Scapim, 2007), y las soluciones de los efectos aleatorios (BLUP) a través del procedimiento MIXED, que puede establecerse en un único proceso iterativo de estimaciónpredicción (Mora y Arnhlod, 2006).

Tanto los componentes de varianza como la predicción de valores genéticos de los árboles forestales pueden ser obtenidos a través de métodos de inferencia Bayesiana (Mora et al., 2007b), cuya metodologia es implementada a través de los Métodos de Monte Carlo Cadenas de Markov (MCMC; del inglés: Markov Chain Monte Carlo). El análisis Bayesiano se basa en el conocimiento de la distribución a posteriori de los parámetros genéticos y viabiliza la conformación de regiones de credibilidad para las estimativas de tales parámetros (Gianola y Fernando, 1986).

Wolfinger y Kass (2000) examinaron regiones de credibilidad para funciones generales de componentes de varianza usando inferencia Bayesiana en un algoritmo de cadena independiente (de la libre traducción de Independence Chain Algorithm). Aqui, la distribución base para los efectos aleatorios es exacta, de modo que las muestras derivadas de los métodos MCMC son rechazadas solamente si no están en el espacio del parámetro, lo cual puede ocurrir, por ejemplo, si un componente de varianza individual es estimado con valor negativo (Mora y Arnhold, 2006). Con ello el algoritmo de cadenas independientes es un caso especial de muestreo de rechazo (Tierney, 1994).

El presente trabajo busca examinar la viabilidad de la obtención de parámetros genéticos y valores predichos en el mejoramiento genético de especies perennes, a través 
de la utilización de una variante de la metodologia MCMC llamado algoritmo de Cadenas Independientes $(\mathrm{Cl})$.

\section{MATERIAL Y MÉTODOS}

\section{Ensayo y Diseño Experimental}

Para ilustrar el análisis genético Bayesiano se utilizó un ensayo de catorce procedencias Australianas de Acacia saligna, establecido en el norte de Chile, en el marco de un proyecto financiado por el Fondo de Desarrollo e Innovación (FDI) y desarrollado por el Instituto Forestal (Perret y Mora, 1999; Mora y Meneses, 2002; Mora et al., 2004).

El ensayo de procedencias fue establecido en la Comunidad Agrícola de Cuz-Cuz, en Illapel, centro norte de Chile $\left(31^{\circ} 63^{\circ} \mathrm{LS} ; 71^{\circ} 22^{\circ}\right.$ LO; ubicación interior con $244 \mathrm{~mm}$ de precipitación media anual), en un diseño de bloques completos al azar, con parcelas de 20 árboles, 3 bloques, y todos los árboles fueron plantados a un distanciamiento de $2 \times 3 \mathrm{~m}$. Mediciones de Diámetro Medio de Copa fueron considerados en el análisis genético de procedencias.

El origen de cada procedencia es mostrado en el Cuadro $\mathrm{N}^{0} 1$.

\section{Cuadro 1}

PROCEDENCIAS DE Acacia saligna CONSIDERADAS EN EL ANALISIS BAYESIANO Y FRECUENTISTA

\begin{tabular}{|l|c|c|c|}
\hline Procedencia & Latitud S & Longitud O & Altitud (m) \\
\hline Ravensthorpe 1 & $33^{\circ} 35^{\circ}$ & $120^{\circ} 08^{\circ}$ & 150 \\
\hline Kelmscott & $32^{\circ} 07^{\prime}$ & $116^{\circ} 02^{\circ}$ & 150 \\
\hline Sanford River & $27^{\circ} 20^{\prime}$ & $116^{\circ} 24^{\circ}$ & 320 \\
\hline Greenough River & $28^{\circ} 42^{\prime}$ & $115^{\circ} 02^{\prime}$ & 175 \\
\hline Geraldton & $28^{\circ} 35^{\prime}$ & $114^{\circ} 37^{\prime}$ & 200 \\
\hline Murchison River & $27^{\circ} 51^{\circ}$ & $114^{\circ} 37^{\circ}$ & 180 \\
\hline Mingenew & $29^{\circ} 12^{\circ}$ & $115^{\circ} 26^{\circ}$ & 180 \\
\hline Lake Indoon & $29^{\circ} 52^{\circ}$ & $115^{\circ} 39^{\circ}$ & 100 \\
\hline Moora & $30^{\circ} 34^{\circ}$ & $116^{\circ} 01^{\circ}$ & 200 \\
\hline Muntadgin Rock & $31^{\circ} 45^{\prime}$ & $118^{\circ} 35^{\circ}$ & 320 \\
\hline Lake Muir & $34^{\circ} 26^{\circ}$ & $116^{\circ} 40^{\circ}$ & 170 \\
\hline Boyatup Hill & $33^{\circ} 14^{\circ}$ & $123^{\circ} 02^{\circ}$ & 183 \\
\hline Lancelin & $31^{\circ} 01^{\circ}$ & $115^{\circ} 20^{\circ}$ & 10 \\
\hline Ravensthorpe 2 & $33^{\circ} 35^{\circ}$ & $120^{\circ} 03^{\circ}$ & 234 \\
\hline
\end{tabular}

\section{REML/BLUP: Análisis de Modelos Mixtos Clásico}

Para la descripción de los valores observados se utilizó el siguiente modelo lineal estadistico, que incluye factores fijos y aleatorios, apropiados para la metodologia de modelos mixtos:

$$
y=X \beta+Z \gamma+\varepsilon
$$


Donde: $y$ es el vector de las respuestas observadas

$X$ es la matriz de diseño de los efectos fijos.

$\beta$ corresponde al vector de parámetros de efectos fijos,

$Z$ es la matriz de incidencia de los efectos aleatorios,

$\gamma$ es el vector de efectos aleatorios de procedencias y

$\varepsilon$ es el vector de residuos.

Se asume que $\gamma$ y $\varepsilon$ son independientes y tienen distribución normal univariada con media 0 y matriz de varianza $G$ y $R$, respectivamente.

Las ecuaciones de modelos mixtos son las siguientes:

$$
\left[\begin{array}{cc}
X^{\prime} R^{-1} X & X^{\prime} R^{-1} Z \\
Z^{\prime} R^{-1} X & Z^{\prime} R^{-1} Z+G^{-1}
\end{array}\right]\left[\begin{array}{c}
\dot{\beta} \\
\dot{\gamma}
\end{array}\right]=\left[\begin{array}{c}
X^{\prime \prime} R^{-1} y \\
Z^{\prime} R^{-1} y
\end{array}\right]
$$

Las ecuaciones descritas de los modelos mixtos, también pueden ser formuladas de la siguiente manera:

$$
\begin{aligned}
& \hat{\beta}=\left(X^{\prime} V^{-1} X\right)^{-} X^{\prime} V^{-1} y \\
& \hat{\gamma}=G Z^{\prime} V^{-1}(y-X \beta)
\end{aligned}
$$

Donde: "-" es una inversa generalizada de la matriz correspondiente,

$V^{-1}$ es la inversa de la matriz de varianza de $y$, definida como $V=Z G Z^{\prime}+R$.

La correlación intraclase fue estimada a partir de la siguiente expresión:

$$
\Gamma_{I c}=\frac{\hat{\sigma}_{p}^{2}}{\hat{\sigma}_{p}^{2}+\hat{\sigma}_{\varepsilon}^{2}}
$$

Donde: $\hat{\sigma}_{p}^{2}$ y $\hat{\sigma}_{e}^{2}$ son los componentes de varianza del efecto de procedencias y residual, respectivamente, estimados vía máxima verosimilitud restringida

(REML).

Las estimaciones de componentes de varianza y las soluciones de los efectos aleatorios fueron calculados en un único proceso iterativo de estimación-predicción, utilizando el procedimiento PROC MIXED de SAS ® 8.02 (SAS Institute, 1996).

\section{Inferencia Bayesiana}

En inferencia Bayesiana, todos los parámetros del modelo son considerados como variables aleatorias (Mora y Arnhold, 2006). Los componentes de varianza, la heredabilidad y los valores genéticos predichos obtenidos por el método Bayesiano se basaron en el promedio, la mediana y la moda de cada muestra a posteriori de tamaño 11000 , generada por el algoritmo de cadena independiente, variante de los métodos MCMC (Markov Chain Monte Carlo). El procedimiento PROC MIXED con opción PRIOR se utilizó para este análisis, construyéndose un conjunto de datos con las informaciones a priori de los parámetros (densidad base). 
De acuerdo al estudio de Mora y Arnhold (2006) y Mora et al. (2007a), se define como el vector de componentes de varianza. La función de densidad conjunta de $(\beta, \gamma, \theta)$ es: $f(\beta, \gamma, \theta[v)=f(\beta, \gamma \mid \theta, y) f(\theta \mid, y)$

La especificación de cada distribución a priori de los elementos del modelo es:

$$
\begin{aligned}
& f\left(1 \mid \beta, \gamma, \sigma_{\varepsilon}^{2}, \sigma_{p}^{2}\right) \sim M(\lambda \beta+Z \gamma, R) \\
& f(\beta) \sim \text { Uniforme } \\
& \left.f\left(\gamma \sigma_{p}^{2}\right) \sim M 0, G\right) \\
& f\left(\sigma_{p}^{2}\right) \sim I G(a, b) \\
& f\left(\sigma_{\varepsilon}^{2}\right) \sim G(c, d)
\end{aligned}
$$

IG corresponde a distribución gamma invertida, con parámetros a priori obtenidos a través del cuadro de densidades base de un análisis preliminar, utilizando distribución no informativa de Jeffreys. La función de densidad a priori del parámetro de efecto fijo es asumida como uniforme (opción FLAT).

La distribución a posteriori conjunta es definida como:

$$
f\left(\beta, \gamma, \sigma_{\varepsilon}^{2}, \sigma_{p}^{2} \mid, v\right) \alpha f(\beta) f\left(\gamma \mid \sigma_{p}^{2}\right) f\left(\sigma_{p}^{2}\right) f\left(\sigma_{\varepsilon}^{2}\right) f\left(\nu / \beta, \gamma, \sigma_{\varepsilon}^{2}, \sigma_{p}^{2}\right.
$$

Las muestras a posteriori fueron analizadas utilizando los procedimientos PROC AUTOREG y UNIVARIATE (SAS Institute, 1996). La moda de cada muestra fue determinada de acuerdo al procedimiento de densidad de Kernel.

\section{RESULTADOS Y DISCUSIÓN}

Los componentes de varianza y la correlación genética intraclase via máxima verosimilitud restringida (REML) e inferencia Bayesiana, basada en el algoritmo de cadenas independientes, son mostrados en el Cuadro $\mathrm{N}^{\circ} 2$. Cabe destacar la inexistencia de periodo de descarte (burn-in) en el algoritmo de cadenas independientes, siendo una ventaja del método al compararlo con los métodos tradicionales de Gibbs (Wolfinger y Kass, 2000).

Los métodos denominados Cadenas de Markov - Monte Carlo (MCMC), permiten el análisis eficiente de complejas superficies de verosimilitud y el cálculo de las distribuciones a posteriori Bayesianas (Mora et al., 2007b). Por esta razón, Walsh (2001) mencionó que en las próximas décadas existirá una fuerte tendencia en el uso de tales procedimientos, reemplazando a su contraparte basada en la función de verosimilitud. El enfoque Bayesiano ha sido también aplicado para examinar la heterogeneidad en muestras genéticas (Roeder et al., 1998), evaluar la diversidad genética (Holsinger, 1999), examinar filogenia (Papini et al., 2007), y en el mapeo de loci de características cuantitativas (QTL; del inglés: Quantitative Trait Loci) (Broman y Speed, 2002).

Los componentes de varianza y correlaciones intraclase fueron muy similares entre ambas metodologias. Tal correlación tuvo un valor de $27,33 \%$ y de $26.99 \%-28,48 \%$, para los procedimientos REML y Bayesiano, respectivamente. George et al. (2006), confirmó una considerable variación genética dentro de poblaciones de Acacia saligna. 
BLUP y algoritmo $\mathrm{Cl}$, entregan valores genéticos similares, no evidenciándose cambios en el ordenamiento de los genotipos (ranking) entre un procedimiento y otro (Cuadro $\mathrm{N}^{\circ} 3$ ). Esto fue confirmado a través de los valores de correlación de Spearman, entre BLUP y cada variable considerada en la metodología Bayesiana, los cuales fueron altos y altamente significativos ( $p<0,01)$ (datos no mostrados). Nogueira et al. (2003), en un análisis comparativo entre inferencia Bayesiana y Frecuentista de un modelo lineal mixto, trabajando con animales encontró valores divergentes entre los componentes de varianza residuales, con altas desviaciones estándar de las varianzas y valores genéticos, utilizando el algoritmo de muestreo de Gibbs, y observó una divergencia importante en el ordenamiento de los genotipos.

En el presente estudio los componentes de varianza son estimados (desconocidos), por lo que, de acuerdo con Littell et al. (1996), la mejor predicción lineal insesgada de los valores genéticos y la mejor estimación lineal no sesgada de los efectos fijos son empíricos, EBLUP (empirical best linear unbiased preditor) y EBLUE (empirical best linear unbiased estimator), respectivamente. Cabe destacar que BLUP es un procedimiento que presenta varias propiedades ventajosas para la predicción genética, el cual es utilizado ampliamente en los programas de evaluación de árboles forestales. Montaldo (2006) menciona las propiedades de BLUP: Mejor (Best) - significa varianza minima de predicción, Lineal (Linear) - significa que las predicciones son funciones lineales de las observaciones, Insesgado (Unbiased) - significa que el valor esperado de las predicciones obtenidos con el modelo lineal son iguales al valor esperado del promedio de los valores genéticos, condicionado a los datos, y Predicción (Prediction) - por que incluye predicción de los valores genéticos aleatorios.

En el presente estudio, ya que los procedimientos de predicción entregan valores genéticos similares, ambas metodologías apuntan como las mejores procedencias a Greenough River, Lancelin y Ravensthorpe 2, indistintamente (Cuadro $N^{\circ} 3$ ). Mora y Perret (2007) mencionan que dentro del contexto del mejoramiento forestal, los métodos Bayesianos pueden ser utilizados eficientemente en la estimación de componentes de varianza, incluyendo la heredabilidad y las correlaciones genéticas, y en la predicción de los valores genéticos.

El análisis Bayesiano es basado en la distribución a posteriori de los parámetros genéticos y viabiliza la conformación de regiones de credibilidad para las estimativas de tales parámetros (Gianola y Fernando, 1986). En el presente estudio, las regiones de credibilidad pueden ser conformadas para cada valor genético, tomando en consideración las desviaciones estándar de cada predicción, las cuales son obtenidas directamente de las distribuciones a posteriori.

El enfoque Bayesiano implementado a través de los diferentes métodos MCMC tal como el muestreo Gibbs, o a través del algoritmo de Cadenas Independientes (como estudiado en el presente artículo), ha mostrado ser apropiado para el análisis tanto de características cuantitativas como categóricas en los programas de mejoramiento (Van Tassell et al., 1998).

La fuerte asociación entre BLUP y el enfoque Bayesiano ha sido ampliamente reportado en la literatura y Blasco (2001) demostró que BLUP es un estimador Bayesiano construido 
usando una información a priori uniforme para los efectos ambientales (en este caso bloques) y una distribución a priori normal para los efectos genéticos.

Finalmente, Mora y Perret (2007) en un estudio similar, pero trabajando también con un modelo de árboles individuales (Animal Model) de eucalipto, destacaron las ventajas de la predicción Bayesiana, ya que lleva en consideración la variabilidad existente en los parámetros genéticos a través del la estimación de las regiones de credibilidad (o intervalos de confianza en el contexto tradicional). Los autores evidenciaron mayores intensidades de selección al utilizar las regiones de credibilidad (Bayesianos) de los efectos genotípicos.

\section{Cuadro $\mathrm{N}^{\circ} 2$}

COMPONENTES DE VARIANZA Y CORRELACIÓN INTRACLASE VIA MAXIMA VEROSIMILITUD RESTRINGIDA (REML) E INFERENCIA BAYESIANA, VÍA ALGORITMO DE CADENAS INDEPENDIENTES

\begin{tabular}{|c|c|c|c|c|c|}
\hline \multirow[t]{2}{*}{ Estimativa } & \multirow[t]{2}{*}{ REML } & \multicolumn{4}{|c|}{$\begin{array}{c}\text { BAYESIANO } \\
\text { (Cadenas Independientes) }\end{array}$} \\
\hline & & Media & Mediana & Moda & $\mathrm{DE}^{1}$ \\
\hline Varianza de Procedencias $\left(\hat{\sigma}_{p}^{2}\right)$ & 438,34 & 480,2657 & 451,5012 & 414,9731 & 168,4781 \\
\hline Varianza Residual $\left(\hat{\sigma}_{\varepsilon}^{2}\right)$ & 1165,35 & 1171,553 & 1166,944 & 1153,097 & 83,18331 \\
\hline Correlación Intraclase $\left(\Gamma_{, c}\right)$ & 0,2733 & 0,2848 & 0,2786 & 0,2699 & 0,06893 \\
\hline
\end{tabular}

'DE: Desviación estándar

Cuadro $\mathrm{N}^{\circ} 3$

VALORES GENÉTICOS DEL EFECTO DE PROCEDENCIAS DE ACACIA, CONSIDERANDO LA MEJOR PREDICCIÓN UNEAR NO SESGADA (BLUP) Y LA INFERENCIA BAYESIANA, VIA ALGORTMO DE CADENAS INDEPENDIENTES

\begin{tabular}{|c|c|c|c|c|c|}
\hline \multirow[t]{2}{*}{ Procedencia } & \multirow[t]{2}{*}{ BLUP } & \multicolumn{4}{|c|}{$\begin{array}{l}\text { PREDICCIÓN BAYESIANA } \\
\text { (Cadenas Independientes) }\end{array}$} \\
\hline & & Media & Mediana & Moda & $\mathrm{DE}^{1}$ \\
\hline Ravensthorpe 1 & $-6,436$ & $-6,545$ & $-6,630$ & $-6,533$ & 9,9 \\
\hline Kelmscott & $-7,768$ & $-7,697$ & $-7,805$ & $-8,481$ & 9,4 \\
\hline Sanford River & 0,444 & 0,580 & 0,515 & 0,680 & 9,6 \\
\hline Greenough River & 14,789 & 14,828 & 14,670 & 13,107 & 9,6 \\
\hline Geraldton & $-14,739$ & $-14,664$ & $-14,645$ & $-15,423$ & 9,4 \\
\hline Murchison River & $-3,829$ & $-3,722$ & $-3,823$ & $-4,573$ & 9,4 \\
\hline Mingenew & $-8,900$ & $-8,751$ & $-8,727$ & $-9,568$ & 9,6 \\
\hline Lake Indoon & $-7,313$ & $-7,127$ & $-7,061$ & $-7,396$ & 9,5 \\
\hline Moora & $-0,032$ & $-0,005$ & $-0,080$ & $-0,888$ & 10,4 \\
\hline Muntadgin Rock & $-6,992$ & $-6,843$ & $-6,937$ & $-7,900$ & 10,3 \\
\hline Lake Muir & $-21,618$ & $-21,558$ & $-21,565$ & $-23,044$ & 10,1 \\
\hline Boyatup Hill & $-18,188$ & $-18,044$ & $-18,036$ & $-19,041$ & 10,1 \\
\hline Lancelin & 34,369 & 34,399 & 34,289 & 35,654 & 9,7 \\
\hline Ravensthorpe 2 & 46,215 & 46,264 & 46,014 & 44,901 & 10,3 \\
\hline
\end{tabular}

'DE: Desviación estándar 


\section{CONCLUSIONES}

La inferencia Bayesiana, vía la variante de los métodos MCMC, cadenas independientes, puede ser utilizada eficientemente en el análisis genético de especies forestales. A la luz de los resultados, se recomienda ampliamente la utilización de inferencia Bayesiana, via este algoritmo, del mismo modo que el análisis clásico de modelos lineales mixtos via REML/BLUP.

\section{REFERENCIAS}

Astorga, M. y F. Mora, 2005. Componentes de Varianza e Interacción Variedad-sitio del Vigor, Producción y Productividad de Olea europaea, en Chile. Cerne 11:25-33.

Blasco, A, 2001. The Bayesian Controversy in Animal Breeding. Journal of Animal Science 79:20232046.

Broman, K.W. and T.P. Speed, 2002. A Model Selection Approach for the Identification of Quantitative Trait loci in Experimental Computing time can be Intensive within each Sweep of Crosses. J R Stat. Soc. 64:641-656.

Gianola, D. and R.L. Fernando, 1986. Bayesian Methods in Animal Breeding Theory. Journal of Animal Science 63:217-244.

George, N., Byrne, M., Maslin, B. and Yan, B., 2006. Genetic Differentiation among Morphological Variants of Acacia saligna (Mimosaceae). Tree Genetics and Genomes 2:109-119.

Holsinger, K.E., 1999. Analysis of Genetic Diversity in Geographically Structured Populations: A Bayesian Perspective. Hereditas 130:245-255.

Littell, R.C., G.A. Milliken, W.W. Stroup, and R.D. Wolfinger, 1996. SAS System for Mixed Models. SAS Institute Inc., Cary, North Caroline, USA. 633 pp.

Montaldo, H.H., 2006. Genetic engineering applications in animal breeding. Electronic Journal of Biotechnology [online]. April 2006, vol. 9, no. 2. Available from Internet: http://www.ejbiotechnology.info/ content/volg/issue $2 /$ full/7/1

Mora, F. y E. Arnhlod, 2006. Inferencia Bayesiana y Metodologia de Modelos Lineales Mixtos Aplicados al Mejoramiento del Maiz. Cien. Inv. Agr. 33:217-223.

Mora, F. y C.A. Scapim, 2007. Predicción de Valores Genéticos del Efecto de Poblaciones de Maíz Evaluadas en Brasil y Paraguay. Agricultura Técnica 67(2):139-146.

Mora, F., O. Pupim-Junior, y C.A. Scapim, 2007a. Predicción del Efecto de Cultivares de Algodón en la Presencia de Interacción Genotipo-Ambiente. Cien. Inv. Agr. 34:11-19.

Mora, F. y S. Perret, 2007. Aplicación de Técnicas Bayesianas en el Análisis Genético de Árboles Forestales. Bosque 28(3).

Mora, F., S. Perret, C.A. Scapim, E.N. Martins, and M.P. Molina, 2007b. Source-dependent Blooming Variability of Eucalyptus cladocalyx in the Region of Coquimbo, Chile. Cien. Inv. Agr. 34(2):99-106. 
Mora, F. y R. Meneses, 2002. Acacia saligna en Zonas Áridas, con Riego más Forraje para el Ganado. Tierra Adentro 47:50-51.

Mora, F., S. Perret y R. Meneses, 2004. Selección de Procedencias de Acacia saligna: Clave para Obtener más Forraje. Tierra Adentro 54:38-40.

Nogueira, D.A., T. Sáfadi, E. Bearzoti and J. S. S. Bueno-Filho, 2003. Análises Clássica e Bayesiana de um Modelo Misto Aplicado ao Melhoramento Animal: Uma llustração. Ciênc. Agrotec. 27:1614-1624.

Papini, A., F. Banci and E. Nardo, 2007. Molecular Evidence of Polyphyletism in the Plant Genus Carum L. (Apiaceae). Genet Mol Biol 30(2):475-482.

Perret, S. y F. Mora, 1999. Acacia saligna: su Impacto en el Norte Chico. Panorama sectorial. Revista Chile Forestal 274:34-36.

Perri, S.H.V. and A.F. lemma, 1999. Procedure of Software SAS® for the Analysis of Mixed Models. Sci. agric. 56(4):959-967.

Roeder, K., M. Escobar and J.B. Kadane, 1998. Measuring Heterogeneity in Forensic Databases using Hierarchical Bayes Models. Biometrika 85:269-287.

SAS INSTITUTE, 1996. Statistical Analysis System: User's Guide. Cary: SAS Institute, 1996. 956p.

Tierney, L., 1994. Markov Chains for Exploring Posterior Distributions (with discussion). Annals of Statistics, 23: $1701-1762$.

Van Tassell, C.P., L.D. Van Vleck and K.E. Gregory, 1998. Bayesian Analysis of Twinning and Ovulation Rates using a Multiple-trait Threshold Model and Gibbs Sampling. Journal of Animal Science 76:20482061.

Wolfinger, R. D. and R. E. Kass, 2000. Non-conjugate Bayesian Analysis of Variance Component Models. Biometrics, 56: 768-774. 